\title{
Plaque Characterization with Computed Tomography Angiography Based on a Diluted-contrast Injection Protocol
}

\author{
Yasuhisa Nakao ${ }^{1}$, Kazuki Yoshida ${ }^{2}$, Shinji Inaba ${ }^{1}$, Yuki Tanabe ${ }^{2}$, Akira Kurata $^{2}$, \\ Teruyoshi Uetani ${ }^{1}$, Teruhito Kido ${ }^{2}$, Katsuji Inoue ${ }^{1}$, \\ Teruhito Mochizuki ${ }^{2}$ and Osamu Yamaguchi ${ }^{1}$
}

\begin{abstract}
:
Objective Coronary plaques with low attenuation on computed tomography (CT) angiography may indicate vulnerable plaques. However, plaque CT attenuation is reported to be significantly affected by intracoronary attenuation. Recently, the diluted-contrast injection protocol was established to facilitate more uniform intracoronary attenuation than can be achieved with the generally used body-weight-adjusted protocol. We validated the relationship between low-attenuation plaque on $\mathrm{CT}$ and lipid-rich plaque using integrated backscatter-intravascular ultrasound (IB-IVUS) as the standard reference.

Methods Plaques were divided into tertiles (T1, T2, and T3) according to the plaque CT attenuation, calculated as the average of five intra-plaque regions of interest, and compared with the plaque characteristics noted on IB-IVUS.

Patients Patients who underwent both CT angiography using a diluted-contrast injection protocol and IBIVUS were retrospectively analyzed.

Results Thirty-nine plaques in 32 patients were analyzed by CT angiography and IB-IVUS. The median plaque CT attenuation (Hounsfield units) of each tertile was 30 (T1), 48 (T2), and 68 (T3). Although no significant difference was noted in conventional quantitative IVUS parameters (e.g. plaque burden), the T1 with lowest plaque CT attenuation had the highest percentage lipid area by IB-IVUS [75.1\% (T1), 57.8\% (T2), and $50.8 \%$ (T3), respectively, $\mathrm{p}<0.01]$. Furthermore, the plaque CT attenuation had a significant negative correlation with the percentage lipid area $(\mathrm{r}=-0.59, \mathrm{p}<0.01)$.

Conclusion CT angiography-based plaque characterization using a diluted-contrast injection protocol may aid in the quantitative detection of lipid-rich plaque.
\end{abstract}

Key words: computed tomography, intravascular ultrasound, coronary artery disease, atherosclerosis, coronary plaque

(Intern Med 60: 3671-3678, 2021)

(DOI: 10.2169/internalmedicine.6683-20)

\section{Introduction}

Computed tomography (CT) angiography is a widely used noninvasive tool for assessing coronary artery stenosis. In addition to facilitating anatomical evaluations, CT has been used increasingly frequently during the last decade for CTbased coronary plaque characterization $(1,2)$. In fact, sev- eral studies have shown that low-attenuation coronary plaque on CT angiography was associated with vulnerable plaque morphology and could predict future cardiovascular events $(3,4)$. However, CT-based plaque characterization is unreliable, as plaque CT attenuation is significantly affected by intracoronary attenuation (5).

Recently, Kawaguchi et al. established a diluted-contrast injection protocol, which affords more homogeneous analy-

${ }^{1}$ Department of Cardiology, Pulmonology, Hypertension and Nephrology, Ehime University Graduate School of Medicine, Japan and ${ }^{2}$ Department of Radiology, Ehime University Graduate School of Medicine, Japan

Received for publication December 15, 2020; Accepted for publication April 13, 2021

Correspondence to Dr. Shinji Inaba, inaba226@gmail.com 
sis of plaque CT attenuation with higher reproducibility than a conventional body-weight-adjusted protocol (6). Previous research groups have shown that integrated backscatterintravascular ultrasound (IB-IVUS) enabled robust tissue characterization of coronary atherosclerotic plaques in in vivo studies $(7,8)$. IB-IVUS provides useful information about plaque characterization associated with cardiovascular events (9-11).

The present study therefore validated the use of an optimized diluted-contrast injection protocol for characterizing coronary plaque using IB-IVUS as a standard reference.

\section{Materials and Methods}

\section{Study population and study protocol}

This was a single-center retrospective observational study for coronary plaques in patients with unstable angina pectoris (UAP) or stable angina pectoris $(\mathrm{SAP})(12,13)$. UAP was defined as the onset of severe, progressive, or resting angina suggestive of an acute coronary syndrome (ACS) coinciding with appropriate objective evidence of myocardial ischemia in coronary angiography, demonstrating 50\% epicardial coronary stenosis. SAP was defined as no change in the frequency, duration, or intensity of chest pain.

We retrospectively analyzed 64 patients who underwent coronary CT angiography using a diluted-contrast injection protocol and IB-IVUS within 3 months of each other between May 2012 and March 2016. The diluted dilutedcontrast injection protocol was clinically implemented as an institutional standard protocol, except in cases with a certain specific cardiovascular disease or history (e.g., post-coronary artery bypass grafting, aortic stenosis before trans-aortic valve intervention). We excluded 32 patients [12 with severe calcified plaques, 9 with no IVUS data before percutaneous coronary intervention (PCI), 5 with in-stent restenosis lesions, 2 with poor CT images due to motion artifacts, 3 with chronic total occlusion (CTO) lesions and 1 with a small coronary plaque lesion]. Ultimately, 32 patients were registered in the present study.

The investigation conforms with the principles outlined in the Declaration of Helsinki and was approved by the research ethics committee of our hospital.

\section{CT angiography using a diluted-contrast injection protocol}

CT angiography was performed by a personalized-contrast injection protocol using the diluted-contrast material (CM) previously reported by Kawaguchi et al. (6). A 256-slice multi-detector row CT scanner (Brilliance iCT; Phillips Healthcare, Cleveland, USA) and an automatic dual injector (Stellant DualFlow; Bayer Yakuhin, Osaka, Japan) were used. At $5 \mathrm{~min}$ prior to the timing bolus scan, $0.6 \mathrm{mg}$ of nitroglycerin spray (Myocor; Astellas Pharma, Tokyo, Japan) was sublingually administered to all patients, and $0.125 \mathrm{mg} /$ $\mathrm{kg}$ of landiolol hydrochloride (Corebeta; Ono Pharmaceuti- cal, Tokyo, Japan) was intravenously administered when the heart rate was $>60$ beats/min. Either iohexol (Omnipaque $350 \mathrm{mg}$ iodine/mL; Daiichi Sankyo, Tokyo, Japan) or iopamidol (Iopamiron $370 \mathrm{mg}$ iodine $/ \mathrm{mL}$; Bayer Yakuhin) was used. The timing bolus scan was performed with $20 \%$ $\mathrm{CM}$ dilution at the same injection rate and volume as that of the main scan $(5 \mathrm{~mL} / \mathrm{s}, 50 \mathrm{~mL})$, followed by a saline chaser $(5 \mathrm{~mL} / \mathrm{s}, 40 \mathrm{~mL}$ ) to estimate the optimal scan timing and the $\mathrm{CM}$ dilution rate to obtain the target arterial attenuation (350 Hounsfield units; HU). The time-attenuation curve (TAC) at the level of the ascending aorta was created with a series of axial CT images. Aortic attenuation was measured at the baseline (before the upslope) and 3 seconds before time-topeak enhancement of the TAC in the timing bolus scan. For the target arterial attenuation of $350 \mathrm{HU}$, the optimal CM dilution rate for the main scan was calculated using the following formula: A $(\%)=20 \% \times(350$-baseline attenuation $) /($ attenuation 3 seconds before time-to-peak enhancement of the TAC-baseline attenuation)

The CM dilution rate was individually calculated using the above two entries in the cardiac CT database. This calculation was automatically performed when the required information was fed into the coronary $\mathrm{CT}$ angiography database, and the optimal CM dilution rate for the real scan was adjusted with an automatic dual injector at intervals of $5 \%$ for clinical use. Subsequently, the main scan was performed with the individually optimized $\mathrm{CM}$ injection protocol (A\%, $5 \mathrm{~mL} / \mathrm{s}$ and $50 \mathrm{~mL}$ ), followed by a saline chaser. When a $\mathrm{CM}$ dilution rate over $100 \%$ was suggested, the injection duration was prolonged according to the increase of the necessary iodine dose at the same injection rate of $5 \mathrm{~mL} / \mathrm{s}$.

Scan parameters were as follows: prospective or retrospective electrocardiogram (ECG) gated scan mode with dose modulation targeting a phase of $75 \% \mathrm{RR}$ interval in ECG at a scan heart rate of $<65$ or $\geq 65$ beats $/ \mathrm{min}$, respectively; tube voltage, $120 \mathrm{kVp}$; effective tube current, 210 (102-210) mAs or 1,064 (800-1,339) mAs/slice; gantry rotation time, 0.27 s/rotation; pitch factor, 0.14 ; collimation, $2 \times$ $128 \times 0.625 \mathrm{~mm}$ with a dynamic $\mathrm{z}$-focal spot, $250 \mathrm{~mm}$ display field of view, and a $512 \times 512$ image matrix.

\section{CT data analyses}

Axial CT data were reconstructed with a slice thickness of $0.8 \mathrm{~mm}$ and a section interval of $0.4 \mathrm{~mm}$ using a medium-smooth cardiac kernel (XCB; Philips Healthcare) and filtered back projection or hybrid iterative reconstruction (iDose level 4; Philips Healthcare). Single CT images with a high image quality at the best phase were selected from the obtained dataset reconstructed in 5\% increments.

CT data analyses were performed by two experienced radiologists (with 5 and 11 years of experience in cardiac imaging) who were blinded to all information of the IB-IVUS. All CT datasets were analyzed with a commercially dedicated workstation (Synapse Vincent; Fuji Medical Systems, Tokyo, Japan). We defined the target plaque in the crosssectional image that presented the minimum lumen area 
A

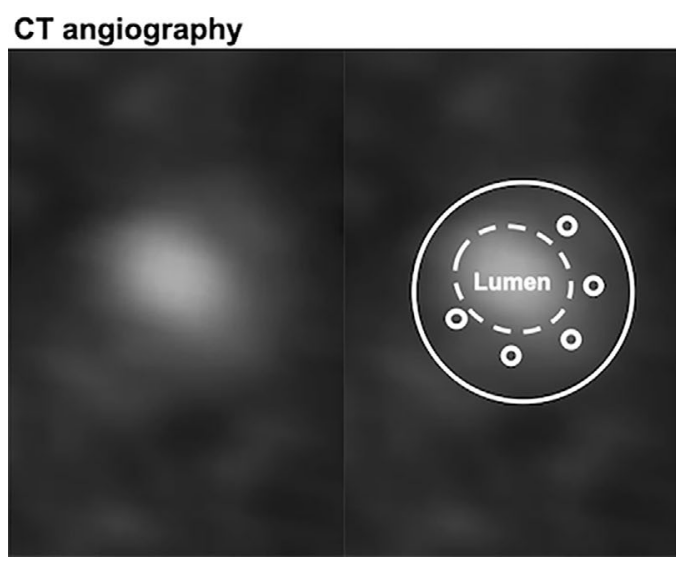

B

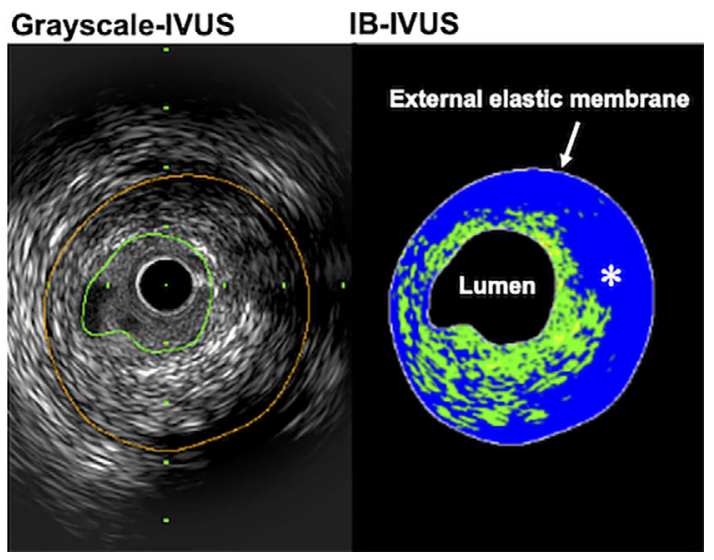

Figure 1. Example of plaque assessments performed by CT angiography and IVUS. A: Cross-sectional image of CT angiography (left panel) using the diluted-contrast injection protocol. Average plaque CT attenuation was calculated with five intra-plaque ROIs (right panel). B: Grayscale IVUS image (left panel) and IB-IVUS image (right panel) corresponding to the CT section. The blue color (asterisk) indicates the large lipid pool, and the green color indicates the fibrous area on IB-IVUS. CT: computed tomography, HU: Hounsfield units, IB: integrated backscatter, IVUS: intravascular ultrasound, ROI: region of interest

(MLA) and calculated the mean attenuation of 5 regions of interest (ROIs; size, 0.2-1.0 $\mathrm{mm}^{2}$ ) placed uniformly around the target plaque as the representative intra-plaque CT attenuation. We defined the normal reference site appearing as a normal vessel in a cross-sectional image proximal to the MLA site. We measured the intracoronary CT attenuation at the normal reference and MLA site by averaging the three ROIs manually placed at the center of the coronary lumen in the cross-sectional images.

\section{IVUS data analyses}

We analyzed IVUS data obtained using a 40-MHz, 5-Fr IVUS imaging catheter (ViewIT; Terumo, Tokyo, Japan) at an automatic pullback of $0.5 \mathrm{~mm} / \mathrm{s}$ using a dedicated software program (VISIATLAS; Terumo).

The cross-sectional area (CSA) of the external elastic membrane (EEM), lumen, plaque and media $(\mathrm{P}+\mathrm{M}$; calculated as EEM-lumen), and plaque burden [calculated as ( $\mathrm{P}+$ $\mathrm{M}) / \mathrm{EEM}$ ] were measured at the MLA site. To ensure that the same coronary sections were compared by IB-IVUS and CT angiography, we first defined the MLA site in coronary CT angiography (CTA) cross-sectional image and identified the anatomical location using the distances from the bifurcation points and side branches as references. In addition, we identified the corresponding coronary sections in IVUS using the reference information by CTA for the frame-level matching carefully. For qualitative IVUS morphologic analyses, plaque attenuation was defined as the absence of an ultrasound signal behind a plaque that was either hypoechoic or isoechoic to the reference adventitia (14), attenuated plaque was defined as plaque with a maximum attenuation angle of $>90^{\circ}$, positive remodeling was defined as CSA of the EEM at MLA site greater than the mean CSA of the ref- erence EEM (15), and spotty calcification was defined as plaque containing only small calcium deposits within a $90^{\circ}$ arc (16).

Coronary plaques in the corresponding sections assessed by IB-IVUS and CT were classified into lipid pool and fibrous by the IB values based on previous IB-IVUS studies $(10,11)$. The percentages of the lipid area and fibrous area were automatically calculated by the IB-IVUS system. An example of a plaque assessment by CT angiography and IVUS is shown in Fig. 1.

\section{Definition of high-risk plaque}

A grayscale-derived high-risk plaque was defined by having at least one of the following known rupture-prone vulnerable plaque morphologies: attenuated plaque, positive remodeling, and spotty calcification. An IB-derived high-risk plaque causing ACS was defined by the presence of a lipid area percentage $>65 \%$, as previously reported (10).

\section{Statistical analyses}

Categorical variables were presented as $\mathrm{n}(\%)$ and continuous variables were expressed as median and interquartile range. The inter-observer reliability in the representative intra-plaque CT attenuation was assessed by interclass correlation coefficients (ICCs). The correlation between the average plaque CT attenuation and percentage of lipid/fibrous area was tested using Spearman's correlation coefficient. Plaques were divided into tertiles based on the average plaque CT attenuation. Categorical variables were compared using Fisher's exact test. Continuous variables were compared using a non-parametric Kruskal-Wallis test, and Bonferroni's test was applied as a follow-up post-hoc analysies. $P$ values $<0.05$ were considered statistically significant. 
Table 1. Baseline Clinical and Lesion Features.

\begin{tabular}{lc}
\hline Patient characteristics (n=32 patients) & \\
Age, years old & $66[60,73]$ \\
Male, sex & $29(91 \%)$ \\
Body weight, $\mathrm{kg}$ & $69[57,76]$ \\
Body mass index, $\mathrm{kg} / \mathrm{m}^{2}$ & $24[22,27]$ \\
Clinical presentation & \\
$\quad$ Stable angina pectoris & $21(66 \%)$ \\
$\quad$ Unstable angina pectoris & $11(34 \%)$ \\
Coronary risk factors & \\
$\quad$ Hypertension & $20(63 \%)$ \\
$\quad$ Diabetes & $12(38 \%)$ \\
$\quad$ Dyslipidemia & $16(50 \%)$ \\
$\quad$ Smoking history & $21(66 \%)$ \\
Family history of coronary artery disease & $11(34 \%)$ \\
& \\
Lesion characteristics (n=39 lesions) & \\
Target vessel & \\
\hline Right coronary artery & $6(15 \%)$ \\
Left anterior descending artery & $28(72 \%)$ \\
Left circumflex artery & $4(10 \%)$ \\
Left main coronary artery & $1(3 \%)$ \\
\hline Values are expressed as median [IQR] or percentage (number of \\
observations/total number of patients).
\end{tabular}

Statistical analyses were performed using EZR (Saitama Medical Center, Jichi Medical University, Saitama, Japan) (17). ICCs were used in the assessment of interobserver reliability for the measurement of the average plaque CT attenuation and calculated using the SPSS software program (version 23.0, SPSS, Chicago, USA).

\section{Results}

\section{Clinical and plaque characteristics}

The clinical and plaque characteristics of 32 patients are shown in Table 1. The median age of the patients was 66 years old, and 91\% were men. Of the 32 patients, 11 (34\%) had an unstable clinical presentation.

\section{CT angiography and IVUS analyses}

CT angiography and IVUS analyses of the 39 plaques are summarized in Table 2. The median value of intracoronary CT attenuation at the proximal portion of the plaque was 348 [314-372] HU, which was very close to the target CT attenuation of $350 \mathrm{HU}$. In contrast, the median value of intracoronary CT attenuation at the MLA site was $278 \mathrm{HU}$. The median CM volume for this protocol was 50 [46-60] $\mathrm{ml}$. The median radiation dose was 8.2 [3.7-14.3] $\mathrm{mSv}$. The ICC for the inter-observer reliability was 0.78 with a $95 \%$ confidence interval of 0.62 to $0.88, \mathrm{p}<0.01$. We found that the reliability was satisfactory (ICC $>0.70$ ).

The relationships between the average plaque CT attenuation and plaque components are shown in Fig. 2. The average plaque $\mathrm{CT}$ attenuation had a significant negative correla- tion with the percentage lipid area by IB-IVUS and a significant positive correlation with the percentage fibrous area by IB-IVUS.

When 39 plaques were divided into tertiles according to the average plaque $\mathrm{CT}$ attenuation, the median average plaque CT attenuation of each tertile was 30, 48, and 68 $\mathrm{HU}$, respectively (Fig. 3A); in contrast, intracoronary CT attenuation at the non-stenotic site was similar among the tertiles (345, 348, and $365 \mathrm{HU}$, respectively) (Fig. 3B). The clinical presentation and IVUS plaque analysis according to the tertile of average CT attenuation are shown in Table 3. Although no significant difference was noted, target plaques responsible for the unstable clinical presentation tended to be most common in the first tertile, followed by the second tertile. A qualitative IVUS analysis showed a significant increase in the frequency of attenuated plaque from the third to first tertiles, along with an increase in the attenuation arc. Similar trends were observed in other vulnerable plaque morphologies (e.g. positive remodeling and spotty calcification). Furthermore, the percentage lipid area measured by IB-IVUS was highest in the first tertile (Fig. 3C). Conversely, the percentage fibrous area measured by IB-IVUS was lowest in the first tertile (Fig. 3D). No significant difference was seen among the tertiles in quantitative IVUS analyses (e.g., EEM CAS, lumen CSA, plaque \& media CSA, and plaque burden).

\section{High-risk plaque}

The frequency of high-risk plaques according to the tertile in the average CT attenuation is shown in Fig. 4. The detection rate of high-risk plaque defined by either grayscale- or IB-IVUS was highest in the first tertile (the detection rate of high-risk plaque in each tertile was $92 \%, 77 \%$, and $31 \%$, respectively, $\mathrm{p}<0.01$ for grayscale IVUS; $77 \%, 31 \%$, and $31 \%$, respectively, $\mathrm{p}<0.05$ for IB-IVUS).

\section{Discussion}

The main findings of the current study are as follows: 1) the CT values of intracoronary attenuation using the dilutedcontrast injection protocol were uniform; 2) the average plaque CT attenuation correlated with grayscale IVUSplaque attenuation; 3) the average plaque CT attenuation correlated with the lipid-rich component on IB-IVUS, although no significant differences in conventional quantitative IVUS parameters were noted; and 4) IVUS-derived high-risk plaques were associated with a low average plaque CT attenuation evaluated by the diluted-contrast injection protocol.

In this study, intracoronary CT attenuation by a dilutedcontrast injection protocol was uniform around the target attenuation. Our data showed that the intra-coronary CT attenuation in the non-stenotic reference site (median $348 \mathrm{HU}$, inter-quantile 314-372 HU) was comparable to that in the previous study by Kawaguchi et al. (346.1 23.9 HU) (6). Therefore, this protocol may have clinical significance for 
Table 2. Computed Tomography Angiography and Intravascular Ultrasound Analysis.

\begin{tabular}{lc}
\hline & Overall 39 lesions \\
\hline Computed tomographic angiography analysis & \\
\hline Average plaque CT attenuation, HU & $48[33,64]$ \\
Intra-coronary CT attenuation, HU (MLA site) & $278[241,335]$ \\
Intra-coronary CT attenuation, HU (proximal of the plaque) & $348[314,372]$ \\
& \\
Intravascular ultrasound analysis at MLA site & \\
Quantitative analysis & \\
EEM CSA, mm ${ }^{2}$ & $12.3[10.0,17.0]$ \\
Lumen CSA, mm ${ }^{2}$ & $2.1[1.8,2.7]$ \\
Plaque \& media CSA, mm ${ }^{2}$ & $9.4[7.7,14.6]$ \\
\%Plaque burden, \% & $82.6[74.4,85.9]$ \\
Qualitative analysis & \\
Plaque attenuation, \% & $17(44 \%)$ \\
Attenuation arc, degree & $82[0,173]$ \\
Positive remodeling, \% & $11(28 \%)$ \\
Spotty calcification, \% & $10(26 \%)$ \\
Lipid area, mm ${ }^{2}$ & $5.9[4.3,7.0]$ \\
Percentage of lipid area, \% & $63.5[49.8,74.6]$ \\
Fibrous area, mm ${ }^{2}$ & $3.3[1.8,4.9]$ \\
Percentage of fibrous area, \% & $31.2[22.7,41.8]$ \\
\hline
\end{tabular}

Values are expressed as median [IQR] or percentage (number of observations/total number of lesions). CSA: cross-sectional area, CT: computed tomography, HU: Hounsfield units, EEM: external elastic membrane, MLA: minimum lumen area
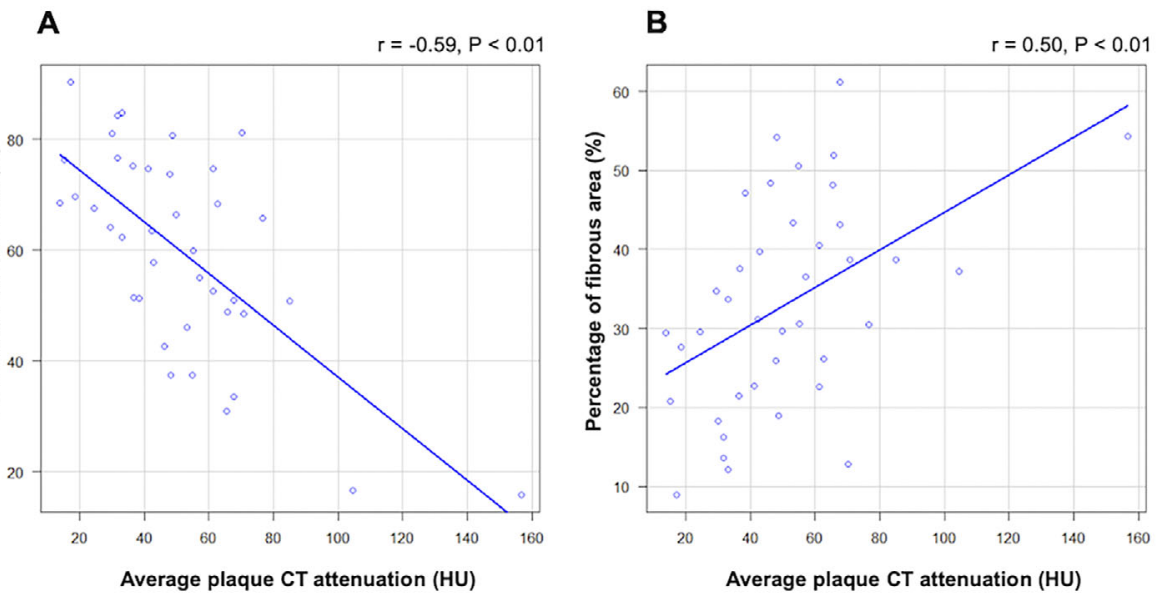

Figure 2. Relationship between the average plaque CT attenuation and plaque components: (A) percentage lipid area and (B) percentage fibrous area. CT: computed tomography, IVUS: intravascular ultrasound

assessing the efficacy of medication on coronary plaque progression and/or regression. Generally, IVUS has been used to evaluate the effects of lipid-lowering therapy on the plaque progression because it has a higher reliability than conventional CT angiography $(18,19)$. Indeed, serial grayscale IVUS studies have shown that statin therapy could reduce coronary plaque volume, regardless of the clinical presentation $(20,21)$. Furthermore, the GLAGOV trial revealed incremental benefits on plaque regression by adding evolocumab, a proprotein convertase subtilisin kexin type 9 inhibitor, to statin treated-patients (22). Furthermore, serial IB-IVUS and near infrared spectroscopy (NIRS)-IVUS studies revealed that even relatively short-term statin therapy could reduce the lipid component, despite no change in the plaque volume $(23,24)$. As a direct link between plaque progression and the clinical outcome was proven by Nicholls et al., evaluating the efficacy of medication on plaque progression has clinical importance (25). However, because IVUS is an invasive approach, the noninvasive assessment of coronary plaque by reproducible CT angiogra- 

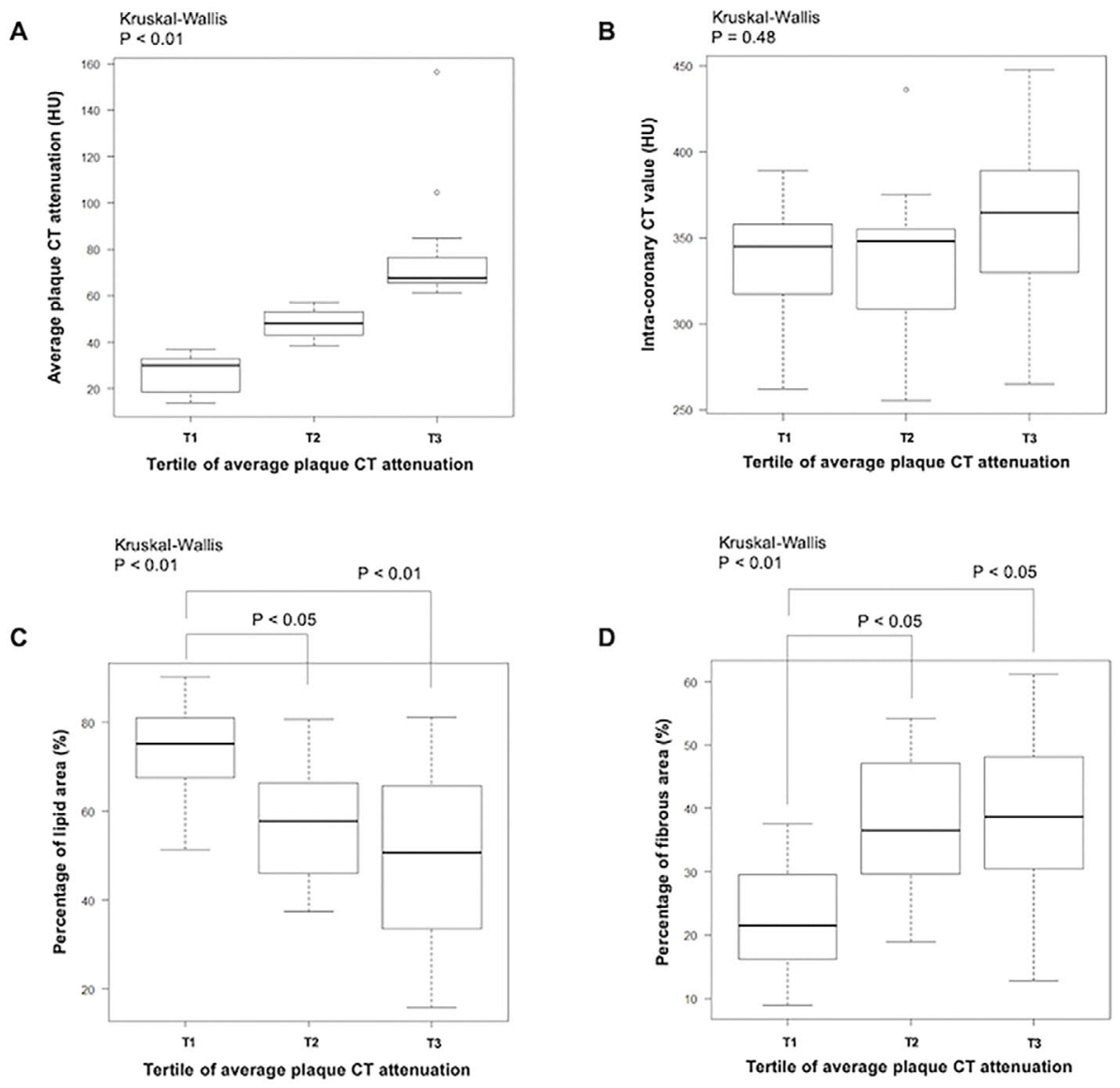

Figure 3. CT angiography analysis results and IB-IVUS findings according to the tertile of average plaque CT attenuation. A: Distribution of average plaque CT attenuation in each tertile. B: A comparison of non-stenotic intracoronary CT attenuation. C, D: A comparison of the percentage lipid area (C) and fibrous area (D). *Box and whiskers plot showing the range and quartiles. CT: computed tomography, IB-IVUS: integrated backscatter-intravascular ultrasound

phy is desirable.

Grayscale IVUS features of plaque attenuation, positive remodeling, and spotty calcification can detect vulnerable plaques responsible for periprocedural myocardial infarction after PCI or future ACS occurrence. Among these features, plaque CT attenuation has shown a correlation with IVUSderived plaque attenuation in the present study. Previous IVUS studies have demonstrated that IVUS-attenuated plaques were associated with ACS presentation and the noreflow phenomenon during PCI $(14,26)$. In addition, a pathological study proved that an IVUS-attenuated plaque was the most reliable surrogate marker of high-risk plaque (27). Similarly, a lipid-rich plaque is also considered a key contributing factor to plaque rupture following ACS events. Sano et al. demonstrated the direct relationship between lipid-rich plaque detected by IB-IVUS and future ACS events (10). In the present study, the lipid component detected by IB-IVUS had a significant negative correlation with plaque CT attenuation. Taken together, our findings suggested that the low plaque CT attenuation using this contrast-injection protocol might be able to detect high-risk plaques responsible for future ACS.

Similarly, Motoyama et al. showed that CT-based low at- tenuation plaque was able to predict future ACS events, and the cut-off attenuation value was $30 \mathrm{HU}$ (4). In addition, Harada et al. reported that plaque characterization by CT might be useful for coronary risk stratification (28). However, there is some concern that the CT attenuation of the plaque might be influenced by ununiform intracoronary iodine attenuation. Therefore, the measurement of plaque CT attenuation with the diluted-contrast injection protocol may be more useful for detecting high-risk plaques than other protocols.

\section{Limitations}

Several limitations associated with the present study warrant mention. First, this study was a retrospective singlecenter study with a relatively small sample size. This might influence the impact of the present findings on clinical practice. Second, the present study included some institution and selection biases. We set the CT-based MLA site and target plaque by frame-level matching and excluded heavily calcified plaques, which are difficult to evaluate on CT. The present results therefore might not reflect plaque characteristics as a whole. Third, the evaluation of plaque CT attenuation is not feasible for heavily calcified plaques, and such assess- 
Table 3. Clinical Presentation and Intravascular Ultrasound Analysis According to Tertile of Average CT Value.

\begin{tabular}{|c|c|c|c|c|}
\hline & Tertile $1(n=13)$ & Tertile $2(n=13)$ & Tertile $3(n=13)$ & $\mathrm{p}$ value \\
\hline \multicolumn{5}{|l|}{ Clinical presentation } \\
\hline Target lesion responsible for unstable clinical presentation & $7(54 \%)$ & $4(31 \%)$ & $2(15 \%)$ & 0.14 \\
\hline \multicolumn{5}{|l|}{ Intravascular ultrasound analysis at MLA site } \\
\hline \multicolumn{5}{|l|}{ Quantitative analysis } \\
\hline EEM CSA, $\mathrm{mm}^{2}$ & $10.8[9.4,17.1]$ & $12.5[11.6,13.0]$ & $11.9[8.3,18.7]$ & 0.80 \\
\hline Lumen CSA, $\mathrm{mm}^{2}$ & $2.0[1.8,2.7]$ & $2.4[2.1,3.2]$ & $1.9[1.8,2.2]$ & 0.18 \\
\hline Plaque \& media CSA, $\mathrm{mm}^{2}$ & $8.8[6.6,14.7]$ & $9.4[8.4,10.9]$ & $9.9[6.2,16.4]$ & 0.92 \\
\hline$\%$ Plaque burden, $\%$ & $81.3[74.5,85.8]$ & $82.6[74.1,84.3]$ & $83.7[75.1,87.7]$ & 0.61 \\
\hline \multicolumn{5}{|l|}{ Qualitative analysis } \\
\hline Plaque attenuation, $\%$ & $10(77 \%)$ & $6(46 \%)$ & $1(8 \%)$ & $<0.01$ \\
\hline Attenuation arc, degree & $149[110,211]$ & $86[0,172]$ & $0[0,0]$ & $<0.01$ \\
\hline Positive remodeling, $\%$ & $5(38 \%)$ & $4(31 \%)$ & $2(15 \%)$ & 0.55 \\
\hline Spotty calcification, $\%$ & $5(38 \%)$ & $3(23 \%)$ & $2(15 \%)$ & 0.54 \\
\hline
\end{tabular}

Values are expressed as median [IQR] or percentage (number of observations/total number of lesions). CSA: cross-sectional area, CT: computed tomography, EEM: external elastic membrane, MLA: minimum lumen area
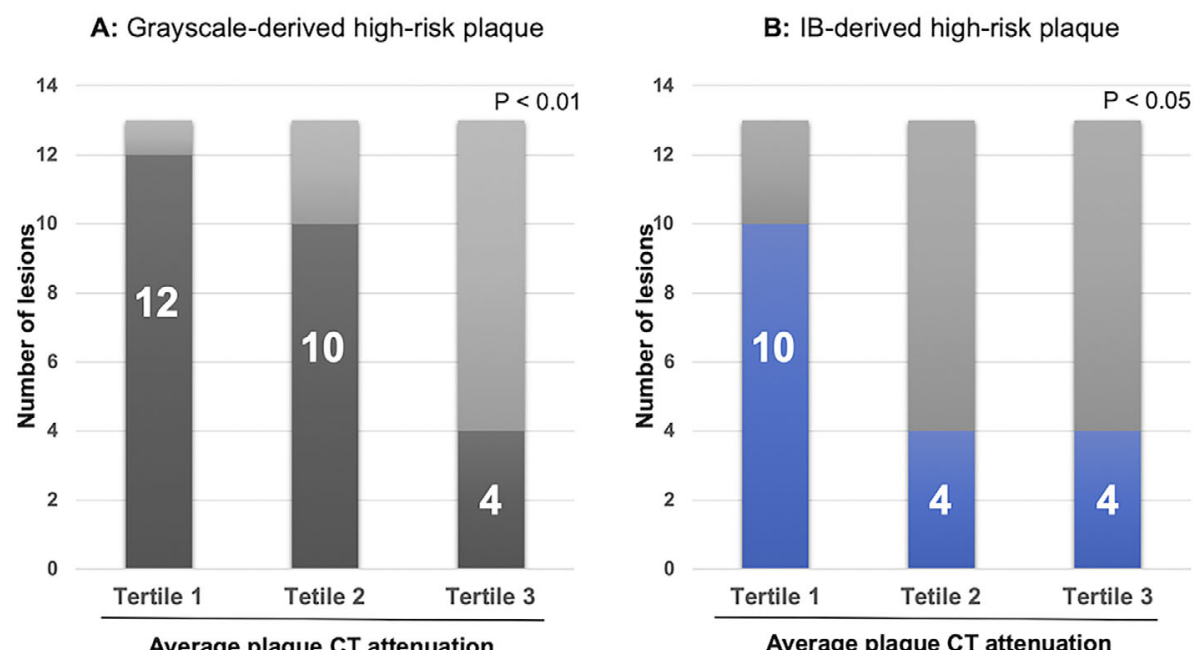

Figure 4. Frequency of high-risk plaque defined by (A) grayscale and (B) IB-IVUS according to the tertile of average plaque CT attenuation. CT: computed tomography, IB-IVUS: integrated backscatter-intravascular ultrasound

ments might not reflect the characteristics of the entire plaque, as the evaluation was only performed at the MLA site. Fourth, given that CT has a limited spatial resolution compared to IVUS, it might be difficult to evaluate distal portions of the plaque. Fifth, we lacked a sufficient number of patients who underwent coronary CT angiography using other contrast injection protocols, since our initial study demonstrated the feasibility of the diluted-contrast injection protocol with regard to the homogeneity of intra-coronary CT attenuation compared to the body-weight-based contrast injection protocol (6). Sixth, there are some concerns about radiation exposure with CT. A 2013 survey in Japan showed that the coronary CT angiography radiation dose was higher than that with routine chest CT (29). The implementation of strategies to reduce CT radiation exposure (e.g. low-tube voltage scanning, retrospective ECG-gated scanning with dose modulation, prospective ECG-gated scanning, and the iterative reconstruction technique) is thus desirable. Finally, we have no direct evidence supporting the relationship between plaque CT attenuation determined by this protocol and future ACS events. To address the issue, longitudinal follow-up studies are needed.

\section{Conclusions}

The diluted-contrast injection protocol allows for robust homogenous CT-based plaque characterization. This protocol may be useful in not only the detection but also the management of high-risk plaques non-invasively.

The authors state that they have no Conflict of Interest (COI).

Yasuhisa Nakao and Kazuki Yoshida contributed equally to this work. 


\section{References}

1. Motoyama S, Kondo $\mathrm{T}$, Anno H, et al. Atherosclerotic plaque characterization by $0.5-\mathrm{mm}$-slice multislice computed tomographic imaging. Circ J 71: 363-366, 2007.

2. Voros S, Rinehart S, Qian Z, et al. Coronary atherosclerosis imaging by coronary CT angiography: current status, correlation with intravascular interrogation and meta-analysis. JACC Cardiovasc Imaging 4: 537-548, 2011.

3. Motoyama S, Kondo T, Sarai M, et al. Multislice computed tomographic characteristics of coronary lesions in acute coronary syndromes. J Am Coll Cardiol 50: 319-326, 2007.

4. Motoyama S, Sarai M, Harigaya H, et al. Computed tomographic angiography characteristics of atherosclerotic plaques subsequently resulting in acute coronary syndrome. J Am Coll Cardiol 54: 4957, 2009.

5. Cademartiri F, Mollet NR, Runza G, et al. Influence of intracoronary attenuation on coronary plaque measurements using multislice computed tomography: observations in an ex vivo model of coronary computed tomography angiography. Eur Radiol 15: 1426-1431, 2005.

6. Kawaguchi N, Kurata A, Kido T, et al. Optimization of coronary attenuation in coronary computed tomography angiography using diluted contrast material. Circ J 78: 662-670, 2014.

7. Ohota M, Kawasaki M, Ismail TF, et al. A histological and clinical comparison of new and conventional integrated backscatter intravascular ultrasound (IB-IVUS). Circ J 76: 1678-1686, 2012.

8. Okubo M, Kawasaki M, Ishihara Y, et al. Development of integrated backscatter intravascular ultrasound for tissue characterization of coronary plaques. Ultrasound Med Biol 34: 655-663, 2008.

9. Kawasaki M, Takatsu H, Noda T, et al. Noninvasive quantitative tissue characterization and two-dimensional color-coded map of human atherosclerotic lesions using ultrasound integrated backscatter: comparison between histology and integrated backscatter images. J Am Coll Cardiol 38: 486-492, 2001.

10. Sano K, Kawasaki M, Ishihara Y, et al. Assessment of vulnerable plaques causing acute coronary syndrome using integrated backscatter intravascular ultrasound. J Am Coll Cardiol 47: 734741, 2006.

11. Ando H, Amano T, Matsubara T, et al. Comparison of tissue characteristics between acute coronary syndrome and stable angina pectoris. An integrated backscatter intravascular ultrasound analysis of culprit and non-culprit lesions. Circ J 75: 383-390, 2011.

12. Ibanez B, James S, Agewall S, et al. 2017 ESC guidelines for the management of acute myocardial infarction in patients presenting with ST-segment elevation. The task force for the management of acute myocardial infarction in patients presenting with ST-segment elevation of the European Society of Cardiology (ESC). Eur Heart J 39: 119-177, 2018.

13. Knuuti J, Wijns W, Saraste A, et al. 2019 ESC guidelines for the diagnosis and management of chronic coronary syndromes. Eur Heart J 41: 407-477, 2020.

14. Lee SY, Mintz GS, Kim SY, et al. Attenuated plaque detected by intravascular ultrasound: clinical, angiographic, and morphologic features and post-percutaneous coronary intervention complications in patients with acute coronary syndromes. JACC Cardiovasc Interv 2: 65-72, 2009.

15. Maehara A, Mintz GS, Bui AB, et al. Morphologic and angiographic features of coronary plaque rupture detected by intravascular ultrasound. J Am Coll Cardiol 40: 904-910, 2002.

16. Ehara S, Kobayashi Y, Yoshiyama M, et al. Spotty calcification typifies the culprit plaque in patients with acute myocardial infarc- tion: an intravascular ultrasound study. Circulation 110: 34243429, 2004.

17. Kanda Y. Investigation of the freely available easy-to-use software 'EZR' for medical statistics. Bone Marrow Transplant 48: 452458, 2013.

18. Nissen SE, Nicholls SJ, Sipahi I, et al. Effect of very highintensity statin therapy on regression of coronary atherosclerosis: the ASTEROID trial. JAMA 295: 1556-1565, 2006.

19. Higashi M. Noninvasive assessment of coronary plaque using multidetector row computed tomography: does MDCT accurately estimate plaque vulnerability? (Con). Circ J 75: 1522-1528, 2011.

20. Hiro $T$, Kimura $T$, Morimoto $T$, et al. Effect of intensive statin therapy on regression of coronary atherosclerosis in patients with acute coronary syndrome: a multicenter randomized trial evaluated by volumetric intravascular ultrasound using pitavastatin versus atorvastatin (JAPAN-ACS [Japan assessment of pitavastatin and atorvastatin in acute coronary syndrome] study). J Am Coll Cardiol 54: 293-302, 2009.

21. Takayama T, Hiro T, Yamagishi M, et al. Effect of rosuvastatin on coronary atheroma in stable coronary artery disease: multicenter coronary atherosclerosis study measuring effects of rosuvastatin using intravascular ultrasound in Japanese subjects (COSMOS). Circ J 73: 2110-2117, 2009.

22. Nicholls SJ, Puri R, Anderson T, et al. Effect of evolocumab on progression of coronary disease in statin-treated patients: the GLAGOV randomized clinical trial. JAMA 316: 2373-2384, 2016.

23. Kawasaki M, Sano K, Okubo M, et al. Volumetric quantitative analysis of tissue characteristics of coronary plaques after statin therapy using three-dimensional integrated backscatter intravascular ultrasound. J Am Coll Cardiol 45: 1946-1953, 2005.

24. Kini AS, Baber U, Kovacic JC, et al. Changes in plaque lipid content after short-term intensive versus standard statin therapy: the YELLOW trial (reduction in yellow plaque by aggressive lipidlowering therapy). J Am Coll Cardiol 62: 21-29, 2013.

25. Nicholls SJ, Hsu A, Wolski $K$, et al. Intravascular ultrasoundderived measures of coronary atherosclerotic plaque burden and clinical outcome. J Am Coll Cardiol 55: 2399-2407, 2010.

26. Wu X, Mintz GS, Xu K, et al. The relationship between attenuated plaque identified by intravascular ultrasound and no-reflow after stenting in acute myocardial infarction: the HORIZONS-AMI (harmonizing outcomes with revascularization and stents in acute myocardial infarction) trial. J Am Coll Cardiol Intv 4: 495-502, 2011.

27. $\mathrm{Pu}$ J, Mintz GS, Biro $\mathrm{S}$, et al. Insights into echo-attenuated plaques, echolucent plaques, and plaques with spotty calcification: novel findings from comparisons among intravascular ultrasound, near-infrared spectroscopy, and pathological histology in 2,294 human coronary artery segments. J Am Coll Cardiol 63: 2220-2233, 2014.

28. Harada K, Amano T, Uetani T, et al. Accuracy of 64-slice multidetector computed tomography for classification and quantitation of coronary plaque: comparison with integrated backscatter intravascular ultrasound. Int J Cardiol 149: 95-101, 2011.

29. Tanabe Y, Kido T, Kimura F, et al. Japanese survey of radiation dose associated with coronary computed tomography angiography - 2013 data from a multicenter registry in daily practice. Circ J 84: 601-608, 2020.

The Internal Medicine is an Open Access journal distributed under the Creative Commons Attribution-NonCommercial-NoDerivatives 4.0 International License. To view the details of this license, please visit (https://creativecommons.org/licenses/ by-nc-nd/4.0/).

(C) 2021 The Japanese Society of Internal Medicine Intern Med 60: 3671-3678, 2021 